\title{
Recruitment, growth and population size structure of Pollicipes pollicipes in SW Portugal
}

\author{
Teresa Cruz ${ }^{\mathrm{a}, \mathrm{b}, *}$, João J. Castro ${ }^{\mathrm{a}, \mathrm{b}}$, Stephen J. Hawkins ${ }^{\mathrm{c}}$ \\ a Laboratório de Ciências do Mar, Universidade de Évora, Apartado 190, 7520-903 Sines, Portugal \\ b Centro de Oceanografia, Faculdade de Ciências da Universidade de Lisboa, Campo Grande, 1749-016 Lisboa, Portugal \\ ' School of Ocean Sciences, Bangor University, Menai Bridge, Anglesey, LL595AB, UK
}

\section{A R T I C L E I N F O}

\section{Keywords:}

Keywords:

Growth

Pollicipes pollicipes

Portugal

Recruitment

Size structure

\begin{abstract}
A B S T R A C T
A general model for the life cycle of Pollicipes pollicipes in SW Portugal is proposed integrating results from a study on recruitment, growth and population size structure. A brief summary of literature concerning Pollicipes published since Margaret Barnes' review paper of 1996 is presented.

Recruitment of $P$. pollicipes on conspecifics (number of cyprids and juveniles with Rostro-Carinal length (RC) $<0.6 \mathrm{~mm}$ per adult) was intense during summer and autumn, but inter-annual variation in the timing and duration of recruitment season was observed. Different indices of recruitment used in Iberian studies were compared. Spatial correlation between shores ( $\sim 40 \mathrm{~km}$ apart) in SW Portugal suggests that cyprids of $P$. pollicipes are being affected by the same larval pool and physical transport processes operating at this scale. Recruitment of $P$. pollicipes in SW Portugal was higher on the low shore ( $\sim 2.5$ times) than on the high shore. Most recruits were attached to the lower half of the adult peduncle. The period of maximum attachment of juveniles ( $\mathrm{RC}<12.5 \mathrm{~mm})$ to conspecifics was summer and autumn (average of 15 juveniles per adult), while spring was the season with a lower number of juveniles growing on adults (average of 2 juveniles). Size of juveniles attached to conspecifics was rarely greater than $6 \mathrm{~mm} \mathrm{RC}$.

Individual annual growth rate of $P$. pollicipes that settled on a denuded surface was $15.7 \mathrm{~mm}$ RC (individuals $\leq$ 1 year old) which corresponds to a monthly increment of $1.3 \mathrm{~mm} \mathrm{RC}$ in their first year of life.

Size structure analyses of $P$. pollicipes attached directly to primary substratum and/or to the base of conspecifics showed that barnacles at the low tide level reached a higher maximum size, indicating that growth at this level was faster than on the high shore. A higher temporal variability between these size-frequency distributions was detected in spring (March to May). In March, two cohorts ( $<1$ year old, $>1$ year old) were identified. In May and August, it was difficult to identify individual different cohorts. From mid autumn to early spring, as a consequence of recruitment of barnacles to the primary substratum, it was again possible to identify two cohorts.
\end{abstract}

(c) 2010 Elsevier B.V. All rights reserved.

\section{Introduction}

Margaret Barnes (1996) published an extensive review on "Pedunculate cirripedes of the genus Pollicipes". Most of this review concerned the European Pollicipes pollicipes and the north eastern Pacific P. polymerus because very little was known about the tropical eastern Pacific P. elegans. More than ten years later, little has been published on P. elegans although several studies have been made on P. pollicipes (e.g. Kugele and Yule, 1996, 2000; Cruz and Hawkins, 1998; Cruz and Araújo, 1999; Molares and Freire, 2003; Macho et al., 2005; Bald et al., 2006; Borja et al., 2006a; Borja et al., 2006b), although much information remains in unpublished Ph.D. theses (e.g. Cruz, 2000; Pavón, 2003; Macho, 2006) (see Table 1). In this paper, we briefly review the literature on Pollicipes published after 1996 (Table 1), and present and

* Corresponding author. Laboratório de Ciências do Mar, Universidade de Évora, Apartado 190, 7520-903 Sines, Portugal. Tel.: +351 269634250; fax: + 351269862057.

E-mail address: tcruz@uevora.pt (T. Cruz). re-analyse information regarding recruitment, growth and population size structure of $P$. pollicipes in SW Portugal originally presented in Cruz (2000).

P. pollicipes is a cirripede distributed in Western Europe and on the North African coasts of the eastern Atlantic from Brittany (France) to Senegal, being rare in the Mediterranean (Barnes, 1996). This species is abundant on very exposed rocky shores, ranging from the shallow subtidal to mid-intertidal zone.

Of all species of the genus Pollicipes, $P$. pollicipes is the most heavily exploited by man. In Spain and Portugal, it is highly prized as food ( $1 \mathrm{~kg}$ can cost up to 150 Euros in Iberian restaurants). P. pollicipes forms clumps of different sizes that are frequently damaged by human harvesting. Considering its vertical intertidal distribution, observations made on the Portuguese coast suggest that exploitation intensity is higher in the low shore than in the mid shore.

$P$. pollicipes is a simultaneous hermaphrodite, breeding more intensively from April to September on SW Portugal (Cruz and Hawkins, 1998). Brooding patterns differ between size classes and are similar 